\title{
Ambulatory surgery in a pediatric patient with polycythemia vera
}

\author{
Claude Abdallah* \\ Anesthesiology and Pediatrics Children's National Health System, 111 Michigan Avenue, Washington D.C, USA
}

\begin{abstract}
Polycythemia vera (PV) is a stem cell disorder characterized as a panhyperplastic and a neoplastic marrow disorder. PV is relatively rare, and extremely uncommon in pediatric patients. The following describes perioperative and anesthetic management of a pediatric patient with polycythemia vera presenting for surgery.
\end{abstract}

\section{Introduction}

Polycythemia vera (PV) is a stem cell disorder characterized as a panhyperplastic and a neoplastic marrow disorder. The most prominent feature of this disease is an elevated absolute red blood cell mass because of uncontrolled red blood cell production. The peak incidence of polycythemia vera (PV) is between ages $50-70$ years. PV is relatively rare, occurring in $0.6-1.6$ persons per million population, and extremely uncommon in pediatric patients, accounting for $0.1 \%$ of all cases [1]. The following is the case of a pediatric patient with polycythemia vera and peripheral cyanosis presenting for elective orodental surgery.

\section{Case presentation}

Patient is an 11 years old female, $46 \mathrm{~kg}$, diagnosed at six years of age with polycythemia vera. Preoperatively, she was alert and interactive, denied any fatigue, chest pain, cough, dyspnea or headaches. Vital signs were: Respiratory rate 18 breaths $/ \mathrm{min}$., pulse $87 / \mathrm{min}$., blood pressure $120 / 75 \mathrm{~mm} \mathrm{Hg}$, temperature $37.2^{\circ} \mathrm{C}$, and oxygen saturation in the $86-88 \%$ range. On exam, she had purple fingernail beds with digital clubbing and erythema of the palms. Breath sounds were clear to auscultation bilaterally. Extremities showed no edema. The family history was significant in that the paternal great grandfather had polycythemia vera. Maternal uncle had a stroke prior to the age of 60 years. A previous echocardiogram showed normal cardiac structures and normal ventricular function with intact atrial and ventricular septum. Complete pulmonary function testing showed mild lower airways obstruction, no restriction and normal diffusion. Chest CT scan showed a prominence of central pulmonary arteries. There was no hepatosplenomegaly on abdominal CT scan. In preparation for surgery, a phlebotomy was performed and hemoglobin (g/dl)/hematocrit (\%) values were brought from 19.8/55.3 to 17.7/49.6. After an inhalation induction with $\mathrm{O}_{2}$ /sevoflurane; fentanyl, propofol and rocuronium were administered intravenously, and the airway was secured with an oral endotracheal tube. Anesthesia was maintained with sevoflurane in an oxygen/air equal mixture. Venodynes were applied throughout the procedure until ambulation in order to prevent deep vein thrombosis. At the end of the procedure, the trachea was extubated and the patient was discharged home after an uneventful recovery.

\section{Discussion}

Several conditions may present or result in elevated red blood cell levels, such as systemic hypoxic conditions, or secondary to hyperstimulation of endogenous erythropoietin secretion, infections or mobilization by secondary causes, such as in leukemoid reactions or thrombocytosis from bleeding and iron deficiency. Physical findings in patients with polycythemia vera are due to manifestations of the myeloproliferative disease. A reddish complexion in the face, palms, nailbeds, mucosa, and conjunctiva is characteristic of PV secondary to the marked increase in total red blood cell mass. Hepatosplenomegaly is often present and due to the manifestations of extramedullary hematopoiesis. This finding is usually associated with hypertension. In the 1970s, the Polycythemia Vera Study Group (PVSG) established the criteria for the diagnosis of PV. The major diagnostic problem is differentiating PV from other more common forms of erythrocytosis. Differential diagnosis includes pseudo-erythrocytosis (decreased plasma volume) which may be eliminated by red blood cell mass measurement and lower levels of erythropoietin in patients with PV. In $34 \%$ of patients with PV, cytogenetic studies show the presence of an abnormal karyotype in the hematopoietic progenitor cells. JAK2 V617F mutation as detected by polymerase chain reaction based methods may represent the first molecular marker for diagnosis of PV. Anesthesia implications are often related to blood hyperviscosity secondary to increase in the cellular components of blood, which impairs microcirculation. Symptoms may include thrombotic events such as arterial or venous thrombosis which result in decreased oxygen delivery and symptoms such as headache, dizziness, vertigo, tinnitus, visual disturbances, claudications, angina, hepatic portal vein thrombosis (Budd-Chiari syndrome) or mesenteric vein thrombosis and stroke. In addition, hyperhomocystinemia is a risk factor for thrombosis and is frequently present in patients with PV (56\%).

Correspondence to: Dr. Claude Abdallah, Assistant Professor of Anesthesiology and Pediatrics Children's National Health System, 111 Michigan Avenue, Washington D.C., USA; Tel: (202) 476-2407; E-mail: cabdalla@cnmc.org

Key words: polycythemia vera, anesthesia, child, perioperative, management

Received: June 16, 2016; Accepted: July 18, 2016; Published: July 21, 2016 
Oxygen content of the arterial blood is the product of the arterial partial pressure of oxygen, the hemoglobin oxygen affinity coefficient, and the hemoglobin concentration. It would seem that the greater the hemoglobin concentration in the blood, the better would be the tissue oxygen delivery. Polycythemia restricts oxygen delivery secondary to its adverse effect on cardiac output as hematocrit increases in result of an elevation in blood viscosity and red blood cell aggregation. In addition, pulmonary vascular resistance increases exponentially with the hematocrit, hypoxemia and polycythemia [2-4]. Bleeding secondary to disruption of hemostatic mechanisms and acquired von Willebrand syndrome may be a complication of PV and presents as ecchymoses, epistaxis, gum or gastrointestinal bleeding. PV is associated with increased histamine levels and gastric acidity which may lead to peptic ulcer disease. Splenomegaly, when present, may cause impaired gastric filling and, rarely, symptoms of splenic infarction. Lastly, pruritus results from increased histamine levels released from increased mast cells and basophils. Anesthesia management should take into consideration all these considerations in order to avoid postoperative complications,
Cardiac output and adequate oxygenation should be well maintained. Achieving hemodynamic stability and preventing acidosis as well as prevention of deep vein thrombosis should be applied. Polycythemia vera with pulmonary arterial hypertension is a rare and potentially life-threatening condition. Scheduling these cases on an ambulatory basis requires a careful preoperative assessment. In the absence of strict guidelines, accepted discharge criteria are individualized and often based on the return to baseline status after an uneventful intra and postoperative course.

\section{References}

1. Berlin NI (1975) Diagnosis and classification of polycythemia. Semin Hematol 12: 339351. [Crossref]

2. McGrath RL, Weil JV (1978) Adverse effects of normovolemic polycythemia and hypoxia on hemodynamics in the dog. Circ Res 43: 793-798. [Crossref]

3. Wells R (1970) Syndromes of hyperviscosity. N Engl J Med. 283: 183-186. [Crossref]

4. Agarwal JB, Paltoo R, Palmer WH (1970) Relative viscosity of blood at varying hematocrits in pulmonary circulation. J Appl Physiol 29: 866-871. [Crossref]

Copyright: (C2016 Abdallah C. This is an open-access article distributed under the terms of the Creative Commons Attribution License, which permits unrestricted use, distribution, and reproduction in any medium, provided the original author and source are credited. 